\title{
ABORDAGEM DO AUTISMO INFANTIL NA ATENÇÃO BÁSICA: REVISÃO INTEGRATIVA
}

\author{
APPROACH TO CHILDHOOD AUTISM IN PRIMARY CARE: \\ INTEGRATIVE REVIEW
}

Nadine Gabryella Pontes Maciel ${ }^{1}$

RESUMO: OBJETIVO: compreender as contribuições da Atenção Básica sobre o Transtorno do Espectro Autista. METODOLOGIA: refere-se a uma revisão integrativa, com o objetivo de reunir resultados de estudos relevantes para a temática. Foi realizado um levantamento de dados bibliográficos nas bases SciELO e Latindex, no período de 2015 a 2017. RESULTADOS: obteve-se um total de oito artigos para análise, onde sete obtiveram destaque por abordar especificadamente a abordagem do Transtorno do Espectro Autista, instituições de acolhimento no âmbito do Sistema Único de Saúde, políticas que resguardam os direitos da pessoa com esse transtorno e a atuação dos profissionais de saúde, destacando a importância da capacitação profissional. CONCLUSÃO: O método escolhido para terapêutica deverá ser realizado envolvendo profissionais da rede capacitados, usuário e família. Destaca-se a importância da Atenção Básica na identificação efetiva e precoce dos sinais do transtorno.

Palavras chave: Atenção básica; Autismo; Saúde pública; Transtornos do espectro autista.

ABSTRACT: OBJECTIVE: To understand the contributions of Primary Care on Autistic Spectrum Disorder. METHODOLOGY: refers to an integrative review, aiming to gather results of studies relevant to the theme. We conducted a survey of bibliographic data in SciELO and Latindex databases, from 2015 to 2017. RESULTS: we obtained a total of eight articles for analysis, where seven were highlighted for specifically addressing the approach of Autistic Spectrum Disorder, institutions of reception within the Unified Health System, policies that safeguard the rights of the person with this disorder and the performance of health professionals, highlighting the importance of professional training. CONCLUSION: The method chosen for therapy should be performed involving trained network professionals, users and

${ }^{1}$ Enfermeira. Pós-graduada em Saúde Pública - UPE. 
families. The importance of primary care in the early and effective identification of signs of the disorder is highlighted.

Keywords: Autism; Primary Care; Public health; Autism Spectrum Disorders. 


\section{INTRODUÇÃO}

O autismo é definido como um transtorno neuropsiquiátrico que apresenta sinais ainda na infância, na maioria dos casos, até os três anos de idade (Zampiroli e Souza, 2012). O Transtorno do Espectro Autista (TEA) é caracterizado por um comprometimento no desenvolvimento, que resulta em comportamentos atípicos nas áreas de interação social, comunicação e comportamento em diferentes graus de comprometimento (Brunoni, Mercadante e Schwartzman, 2014).

As principais características do TEA envolve o desenvolvimento incomum da linguagem e comunicação, falha na interação com o meio social, ineficácia na demonstração de aspectos emocionais, além de apresentar uma estima a atividades restritas e repetitivas (Camargo e Bosa, 2009). Geralmente, os pais percebem algo errado com seus filhos, podendo achar que ele é surdo, uma vez que não responde a comandos e estímulos (Klin, 2006).

Estudos comprovam um aumento considerável na prevalência do TEA, por mais que existam deficiências no diagnóstico, o que demonstra a relevância do assunto para a Saúde Pública (Defense-Netvral e Fernandes, 2016). Segundo a Organização Pan-Americana de Saúde (2017), estima que uma a cada 160 crianças no mundo tem TEA.

A disseminação do conteúdo e a expansão de critérios diagnósticos são algumas das justificativas para o aumento alarmante no número de casos nos últimos anos. Vale ressaltar que os dados epidemiológicos contribuem e são fundamentais para a elaboração, aplicação e ampliação de políticas que envolvem a população autista, destacando assim a importância no investimento de pesquisas voltadas para essa área.

A Política Nacional de Atenção Básica traz consigo a valorização do sujeito, singularidade e inserção social, atendendo as necessidades da população, sem nenhuma distinção (Oliveira, Feldman, Couto e Lima, 2017). As Redes de Atenção à Saúde (RAS) buscam melhorar a atenção por meio da expansão do acesso, 
objetivando a integralidade e o atendimento a ocorrências agudas e condições crônicas de saúde, tendo a Atenção Básica como o centro para a ordenação do cuidado (Peiter, Santos, Lanzoni, Mello, Costa e Andrade, 2019).

É de suma importância o manejo de crianças com TEA na Atenção Básica $(A B)$ de forma integral e a articulada em rede, objetivando as necessidades do usuário e família, ofertando um atendimento multiprofissional, uma vez que a integralidade na prática do cuidado associa-se à superação do olhar centrado na problemática.

O objetivo deste trabalho é compreender, através de uma revisão integrativa da literatura, o Transtorno do Espectro Autista, o autismo infantil e as contribuições da $A B$ sobre esse transtorno. Considera-se que os resultados apresentados neste trabalho poderão contribuir para a melhoria da assistência e na construção de conhecimento dos profissionais de saúde atuantes na $A B$.

\section{METODOLOGIA}

Trata-se de uma revisão integrativa, que tem o objetivo reunir resultados de estudos desenvolvidos conforme diversas metodologias, permitindo aos revisores unificar resultados sem alterar estudos envolvidos (Soares, Hoga, Peduzzi, Sangaleti, Yonekura e Silva, 2014). Sua relevância consistiu-se na síntese de múltiplos estudos com a perspectiva de oferecer subsídios e orientações para as mudanças e melhorias na qualidade das ações assistenciais da saúde.

$\mathrm{Na}$ elaboração da pesquisa, forem percorridas seis fases: na primeira foi definida a pergunta norteadora, na segunda foi realizada uma busca e amostragem da literatura, na terceira foi realizada a coleta de dados, na quarta etapa foram feitas análises críticas dos estudos incluídos, na quinta etapa foi desenvolvida a discussão dos resultados e na sexta etapa foi apresentada a finalização da revisão. A questão norteadora determinada foi: "Qual a abordagem da Atenção Básica para com as crianças diagnosticadas ou com sinais precursores do autismo?" 
Para o levantamento bibliográfico, foram realizadas buscas de estudos nas bases de dados Scientific Electronic Library Online - SciELO e Sistema Regional de Información em Línea para Revistas Científicas de América Latina, el Caribe, España y Pertugal - Latindex, empregando os descritores em português. Os critérios de inclusão foram artigos que atendessem a temática da pesquisa, publicados no período de 2015 a 2017, intervalo que se destacou por apresentar um maior número de estudos relevantes para o contexto. O critério de exclusão foram estudos que não condiziam com a proposta do tema, além de publicações que se repetiam nas bases de dados. A busca foi realizada no primeiro semestre de 2019, utilizando os descritores "Saúde Pública", "Atenção Básica”, "Transtornos do Espectro Autista" e "Autismo" padronizados para busca, e disponíveis nos Descritores em Ciências da Saúde (DeCS).

Para formulação da revisão verificou-se inicialmente os artigos por títulos, posteriormente a leitura dos resumos e por fim a leitura na íntegra do artigo. Foram identificados 40 estudos na base de dados SciELO e 15 artigos na base de dados Latindex, de acordo com as combinações dos descritores. Ao final, foram utilizados 8 artigos. A coleta de dados foi realizada durante 3 meses, com início em Janeiro de 2019 e término em Abril de 2019. Os trabalhos foram classificados e agrupados por assunto, autores e ano de publicação, objetivo do artigo, tipologia do estudo e principais resultados. O esquema da busca e número de estudos encontrados e escolhidos conforme critérios de inclusão e exclusão, está presente na Figura 1. 


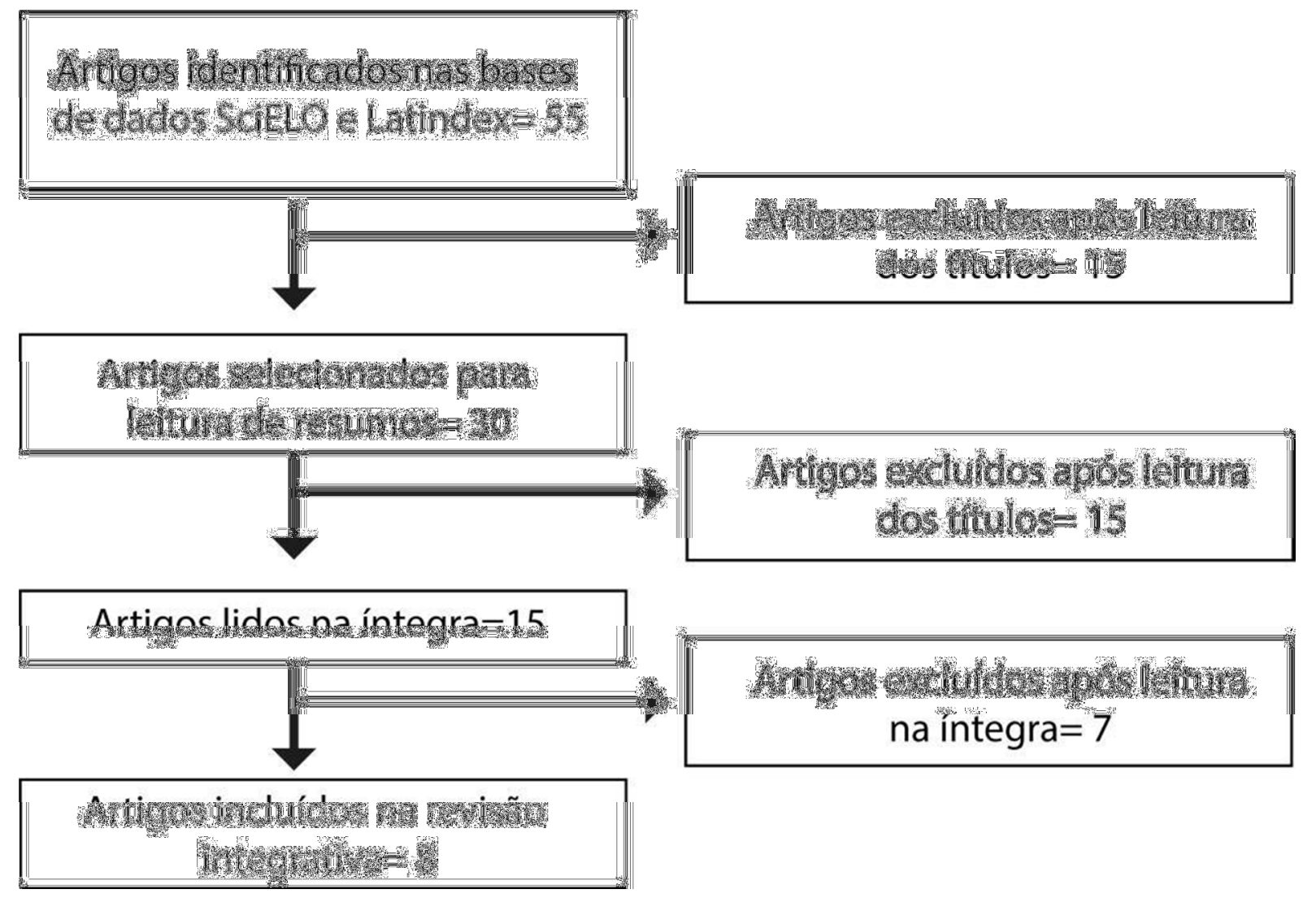

Figura 1. Esquema de busca de estudos escolhidos para a revisão integrativa.

\section{RESULTADOS E DISCUSSÃO}

Foram avaliados 8 artigos, publicados distribuídos em periódicos de circulação nacional e internacional. Na Tabela 1 serão apresentados os principais resultados desta revisão integrativa, com a caracterização das publicações e o conteúdo dos artigos analisados. 
Tabela 1. Caracterização dos artigos segundo Qualis, assunto, autores e ano de publicação, objetivo da pesquisa, tipologia do estudo e principais resultados.

\begin{tabular}{|c|c|c|c|c|c|}
\hline Qualis & Assunto & Autor/Ano & $\begin{array}{l}\text { Objetivo do } \\
\text { artigo }\end{array}$ & $\begin{array}{c}\text { Tipologia do } \\
\text { estudo }\end{array}$ & Principais resultados \\
\hline B1 & $\begin{array}{l}\text { A Produção } \\
\text { Científica } \\
\text { Brasileira } \\
\text { sobre } \\
\text { Autismo na } \\
\text { Psicologia e } \\
\text { na Educação }\end{array}$ & $\begin{array}{l}\text { GUEDES e } \\
\text { TADA (2015) }\end{array}$ & $\begin{array}{l}\text { Identificar o } \\
\text { atual cenário } \\
\text { da produção } \\
\text { científica sobre } \\
\text { a temática do } \\
\text { autismo }\end{array}$ & $\begin{array}{l}\text { Revisão de } \\
\text { Literatura }\end{array}$ & $\begin{array}{l}\text { Identificou-se que, em todas as } \\
\text { categorias, esteve presente a } \\
\text { sistematização das } \\
\text { características do autismo ea } \\
\text { elaboração de intervenções em } \\
\text { prol da diminuição dos sintomas. }\end{array}$ \\
\hline $\mathrm{C}$ & $\begin{array}{c}\text { A importância } \\
\text { da família no } \\
\text { cuidado da } \\
\text { criança } \\
\text { autista }\end{array}$ & $\begin{array}{l}\text { MAIA FILHO, } \\
\text { MORAIS, } \\
\text { SILVA e } \\
\text { SANTIAGO } \\
(2016)\end{array}$ & $\begin{array}{l}\text { Análise da } \\
\text { participação da } \\
\text { família no } \\
\text { cuidado da } \\
\text { criança autista }\end{array}$ & $\begin{array}{l}\text { Abordagem } \\
\text { qualitativa } \\
\text { com } \\
\text { delineamento } \\
\text { descritivo } \\
\text { exploratório }\end{array}$ & $\begin{array}{l}\text { O estudo mostrou que a família } \\
\text { não está preparada para cuida } \\
\text { de uma criança autista, pois é } \\
\text { uma situação inesperada no } \\
\text { contexto familiar. }\end{array}$ \\
\hline B1 & $\begin{array}{c}\text { A oferta da } \\
\text { terapia } \\
\text { fonoaudiológi } \\
\text { ca em locais } \\
\text { de assistência } \\
\text { a indivíduos } \\
\text { com } \\
\text { Transtornos } \\
\text { do Espectro } \\
\text { do Autista } \\
\text { (TEA) }\end{array}$ & $\begin{array}{l}\text { DEFENSE- } \\
\text { NETRVAL } \\
\text { FERNANDES } \\
(2016)\end{array}$ & $\begin{array}{l}\text { Caracterizar a } \\
\text { oferta desse } \\
\text { serviço nos } \\
\text { diversos locais } \\
\text { de assistência a } \\
\text { essa população } \\
\text { no município } \\
\text { de São Paulo }\end{array}$ & $\begin{array}{l}\text { Abordagem } \\
\text { qualitativa e } \\
\text { quantitativa }\end{array}$ & $\begin{array}{l}\text { Observou-se que apenas } 64 \% \\
\text { dos locais oferecem o } \\
\text { acompanhamento } \\
\text { fonoaudiológico e que o número } \\
\text { dos indivíduos com indicação } \\
\text { para esse acompanhamento está } \\
\text { aquém do esperado }\end{array}$ \\
\hline B1 & $\begin{array}{c}\text { Atenção } \\
\text { psicossocial a } \\
\text { crianças e } \\
\text { adolescentes } \\
\text { com autismo } \\
\text { nos CAPSi da } \\
\text { região } \\
\text { metropolitana } \\
\text { do Rio de } \\
\text { Janeiro }\end{array}$ & $\begin{array}{l}\text { LIMA, COUTO, } \\
\text { SOLIS, } \\
\text { OLIVEIRA e } \\
\text { DELGADO } \\
\text { (2017) } \\
\end{array}$ & $\begin{array}{l}\text { Apresentar e } \\
\text { discutir dados } \\
\text { gerais a } \\
\text { respeito do } \\
\text { atendimento } \\
\text { oferecido a } \\
\text { crianças e } \\
\text { adolescentes } \\
\text { com autismo } \\
\text { nos CAPSi da } \\
\text { região } \\
\text { metropolitana } \\
\text { do Rio de } \\
\text { Janeiro, além } \\
\text { de dados sobre } \\
\text { o perfil } \\
\text { psicossocial } \\
\text { dessa } \\
\text { população }\end{array}$ & $\begin{array}{c}\text { Estudo } \\
\text { descritivo, } \\
\text { baseado em } \\
\text { dados } \\
\text { quantitativos } \\
\\
\end{array}$ & $\begin{array}{c}\text { Havia } 782 \text { usuários com } \\
\text { diagnóstico de autismo nos } \\
\text { CAPSi pesquisados, constituindo } \\
1 / 3 \text { dos usuários em } \\
\text { atendimento. A maioria era do } \\
\text { sexo masculino, na faixa dos } 10 \\
\text { aos } 19 \text { anos. A maior parte } \\
\text { residia com a família, na área de } \\
\text { abrangência do serviço, e estava } \\
\text { em regime assistencial semi- } \\
\text { intensivo, com atendimentos } \\
\text { individuais e coletivos, incluindo } \\
\text { os familiares. Cerca de } 1 / 5 \\
\text { estava fora da escola e menos } \\
\text { de } 1 / 3 \text { recebia o Benefício de } \\
\text { Prestação Continuada da Lei } \\
\text { Orgânica da Assistência Social } \\
\text { (BPC -Loas) }\end{array}$ \\
\hline
\end{tabular}




\begin{tabular}{|c|c|c|c|c|c|}
\hline B4 & $\begin{array}{c}\text { Autismo } \\
\text { infantil: } \\
\text { acolhimento e } \\
\text { tratamento } \\
\text { pelo Sistema } \\
\text { Único de } \\
\text { Saúde }\end{array}$ & $\begin{array}{l}\text { NASCIMENTO, } \\
\text { PEREIRA e } \\
\text { GARCIA (2017) }\end{array}$ & $\begin{array}{c}\text { Apresentar o } \\
\text { diagnóstico do } \\
\text { Transtorno do } \\
\text { Espectro } \\
\text { Autista em } \\
\text { crianças, o } \\
\text { acolhimento } \\
\text { oferecido à } \\
\text { família e ao } \\
\text { tratamento } \\
\text { multidisciplinar } \\
\text { oferecidos pelo } \\
\text { SUS - Sistema } \\
\text { Único de } \\
\text { Saúde, em } \\
\text { âmbito } \\
\text { nacional. }\end{array}$ & $\begin{array}{l}\text { Revisão de } \\
\text { literatura }\end{array}$ & $\begin{array}{l}\text { O autismo caracteriza-se pelo } \\
\text { desenvolvimento atípico e } \\
\text { inadequado das linguagens e } \\
\text { comunicação, meio social e } \\
\text { emocional, além de apresentar } \\
\text { interesses e atividades restritas. } \\
\text { Devido ao crescente aumento do } \\
\text { diagnóstico de transtornos } \\
\text { neuropsiquiátricos, torna-se } \\
\text { necessário que profissionais em } \\
\text { todas as áreas da saúde atuem } \\
\text { em equipe, para um completo } \\
\text { amparo à criança e sua família. }\end{array}$ \\
\hline B1 & $\begin{array}{c}\text { Importância } \\
\text { do } \\
\text { acolhimento } \\
\text { de pais que } \\
\text { tiveram } \\
\text { diagnóstico } \\
\text { do transtorno } \\
\text { do espectro } \\
\text { do autismo de } \\
\text { um filho }\end{array}$ & $\begin{array}{c}\text { MAIA, } \\
\text { ALMEIDA, } \\
\text { OLIVEIRA, } \\
\text { OLIVEIRA, } \\
\text { SAEGER, } \\
\text { OLIVEIRA e } \\
\text { SILVEIRA } \\
(2016)\end{array}$ & $\begin{array}{l}\text { Avaliar os } \\
\text { resultados de } \\
\text { uma } \\
\text { capacitação } \\
\text { oferecida aos } \\
\text { membros de } \\
\text { uma equipe de } \\
\text { acolhimento de } \\
\text { pais cujo } \\
\text { filho(a) teve } \\
\text { diagnóstico de } \\
\text { transtorno de } \\
\text { espectro do } \\
\text { autismo (TEA). }\end{array}$ & $\begin{array}{c}\text { Pesquisa } \\
\text { exploratória } \\
\text { com } \\
\text { abordagem } \\
\text { qualitativa }\end{array}$ & $\begin{array}{l}\text { Observou-se a mudança de } \\
\text { percepção na assertiva que } \\
\text { avaliava a necessidade de se } \\
\text { compreender a importância do } \\
\text { acolhimento de pais de crianças } \\
\text { com TEA. Verificou-se que, } \\
\text { antes da capacitação, os } \\
\text { participantes apresentavam um } \\
\text { envolvimento emocional com o } \\
\text { trabalho a ser desenvolvido no } \\
\text { acolhimento; após a capacitação, } \\
\text { eles deram ênfase à importancia } \\
\text { de orientar os pais na busca por } \\
\text { profissionais qualificados e à } \\
\text { importância do } \\
\text { acompanhamento da criança por } \\
\text { uma equipe multiprofissional. A } \\
\text { escuta também foi referida antes } \\
\text { e após a capacitação }\end{array}$ \\
\hline B4 & $\begin{array}{c}\text { Mapeamento } \\
\text { dos serviços } \\
\text { que prestam } \\
\text { atendimento a } \\
\text { pessoas com } \\
\text { transtorno do } \\
\text { espectro } \\
\text { autista no } \\
\text { Brasil }\end{array}$ & $\begin{array}{l}\text { PORTOLESE, } \\
\text { BORDINI, } \\
\text { LOWENTHAL, } \\
\text { ZACHI e de } \\
\text { PAULA (2017) }\end{array}$ & $\begin{array}{c}\text { Mapear as } \\
\text { instituições } \\
\text { brasileiras que } \\
\text { prestam } \\
\text { atendimento a } \\
\text { indivíduos com } \\
\text { TEA }\end{array}$ & $\begin{array}{c}\text { Abordagem } \\
\text { qualitativa e } \\
\text { quantitativa } \\
\text { com TEA }\end{array}$ & $\begin{array}{c}\text { Verificaram-se } 650 \text { instituições, } \\
\text { das quais a maioria concentra-se } \\
\text { nas regiões Sudeste e Sul A } \\
\text { maior parte consiste nas } \\
\text { Associações de Pais Amigos } \\
\text { dos Excepcionais-APAEs, } \\
\text { seguidas pelos Centros de } \\
\text { Atenção Psicossocial Infanto- } \\
\text { juvenil-CAPSls e AMAs. Há } \\
\text { predominância do atendimento à } \\
\text { infância e adolescência. O } \\
\text { atendimento multiprofissional foi } \\
\text { mais frequente e dentre as } \\
\text { abordagens teóricas destacam- } \\
\text { se os métodos TEACCH, } \\
\text { Psicoeducação e ABA. }\end{array}$ \\
\hline
\end{tabular}




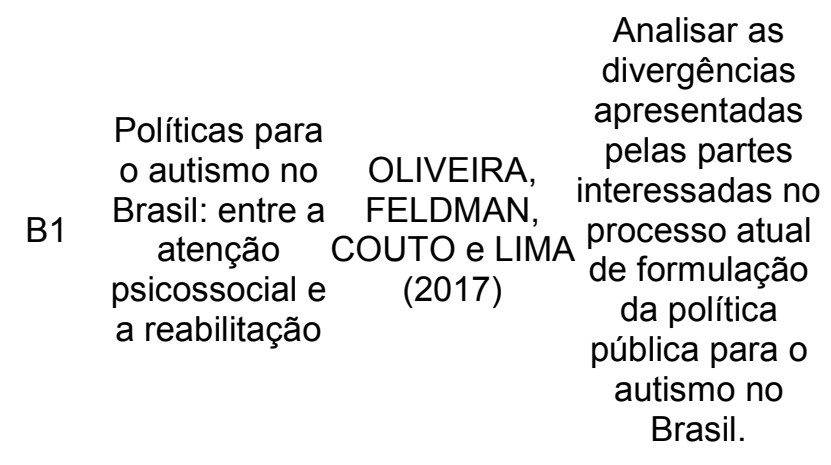

Embora os dissensos teóricos e clínicos representem um importante fator nas controvérsias em questão, estes não aparentam compor o elemento principal do conflito, uma vez que se mostram negociáveis. As discordâncias

Análise documental parecem se concentrar em torno de uma reivindicação, pelos grupos envolvidos, acerca de qual deles seria detentor de maior expertise e legitimação em relação ao autismo, como forma de reconhecê-los como atoreschave na proposição da política pública.

Dos oito artigos selecionados, sete $(87,5 \%)$ se destacaram por abordar especificadamente a abordagem do TEA, as instituições de acolhimento no âmbito do SUS, as políticas existentes que resguardam os direitos da pessoa com TEA e a atuação dos profissionais de saúde, destacando a importância da capacitação profissional. Dois artigos (25\%) discutiram sobre a importância da participação da família no cuidado da criança diagnosticada com TEA. Quanto a metodologia, três $(37,5 \%)$ estudos utilizaram a abordagem qualitativa e quantitativa, dois (25\%) estudos utilizaram o tipo revisão de literatura, dois (25\%) estudos realizaram uma pesquisa exploratória com abordagem qualitativa e um (12,5\%) estudo empregou a análise documental.

\section{Abordagem do autismo infantil na atenção básica}

Defense-Netrval e Fernandes (2016) e Lima et al. (2017) afirmam que a prioridade para no tratamento dos casos de TEA é a precoce efetivação diagnóstica e o rápido início da terapêutica, aumentando as chances de um melhor prognóstico.

Maia Filho et al. (2016) relata que para auxiliar os profissionais na avaliação e diagnóstico de diversos transtornos, a Associação Psiquiátrica Americana formulou um documento difundido mundialmente, chamado de Manual Diagnóstico e Estatístico de Transtornos Mentais (DSM). 
Outra classificação de diagnóstico utilizada mundialmente é o CID 11 (Classificação Estatística Internacional de Doenças e Problemas Relacionados à Saúde) definido pela OMS (Organização Mundial da Saúde), onde o autismo se adequa nos Transtornos mentais, comportamentais ou do neurodesenvolvimento, (Organização Pan-Americana de Saúde, 2018).

Guedes e Tada (2015) relatam que o diagnóstico do autismo não é de fácil realização, sendo feito em grande parte, através de avaliação clínica do comportamento em conjunto com os critérios clínicos contidos no CID-11 e DSM-V. O fato da criança, conforme seu crescimento e amadurecimento, não apresentar interações que os pais tencionam, ocasiona uma suspeita sobre alguma alteração ou deficiência que a criança possa vir a ter, iniciando uma luta extensa para descobrir o motivo de tal situação (Maia Filho et al., 2016).

Não se tem conhecimento de exames que confirmem o diagnóstico do TEA, porém alguns procedimentos podem ser feitos para exclusão de transtornos que apresentam uma sintomatologia semelhante ao TEA, trazendo mais segurança na determinação do diagnóstico (Guedes e Tada, 2015).

Ainda que se tenha documentos que sirvam como base para o diagnóstico, a realização do mesmo não é simples, requerendo um olhar clinico minucioso e precoce, pois quanto mais cedo for feita a identificação de traços autísticos, mais fácil será a reabilitação e menos atribulado será o prognóstico da pessoa com TEA.

Para a $A B$, formada por Unidades Básicas de Saúde e de Equipes de Estratégia de Saúde da Família, é atribuída a função de identificar precocemente os casos de TEA, o acompanhamento e a assistência integral aos usuários (Portolese et al., 2017).

Guedes e Tada (2015) relatam que conforme observadas as necessidades da pessoa portadora do autismo, condições associadas a ele tem sido acobertadas por políticas sociais, levantando questões que envolvem a rápida identificação e o tipo e intensidade dos tratamentos.

Segundo Defense-Netvral e Fernandes (2016) somente sete estados no Brasil possuem políticas públicas voltadas ao TEA, o que revela uma dificuldade na consideração e inclusão do autismo como uma deficiência no país. 
A partir de 2013, após observar as necessidades da população, o Ministério da Saúde lançou dois guias de trabalho para o atendimento dos casos de TEA no âmbito do SUS, sendo o primeiro as Diretrizes de atenção e reabilitação para pessoas com TEA autismo e a segunda a Linha de cuidado para a atenção às pessoas com transtornos do espectro do autismo e suas famílias na Rede de Atenção Psicossocial do SUS (Defense-Netrval e Fernandes, 2016).

Através da Lei $n^{0} 12.764$, de 27 de dezembro de 2012, foi instituída a Política Nacional de Proteção dos Direitos da Pessoa com TEA, que além de trazer artigos que definem o que é o TEA, a consideração de que o indivíduo com TEA é uma pessoa com deficiência, para todos os efeitos legais, destaca também a garantia dos direitos que envolve uma vida de qualidade, com a manutenção da integridade física e moral, segurança, lazer e acesso a ações e serviços de saúde, que atendam integralmente as suas necessidades, como o diagnóstico precoce, ainda que não conclusivo, o atendimento multiprofissional, terapia nutricional e medicamentos (Defense-Netrval e Fernandes, 2016).

Maia Filho et al. (2016) e Maia et al. (2016) apontam que o diagnóstico é muito importante e gera impasses na família, ressaltando ainda que em nosso país houve um avanço no diagnóstico do autismo, porém não são observados relatos de como essa família foi recebida após diagnóstico, um momento delicado, que pode facilitar o enfrentamento da situação.

Para otimizar a organização e atendimento, o SUS foi categorizado e hierarquizado em níveis primário, secundário e terciário, conforme os níveis de complexidade, onde o setor primário é considerada a porta de entrada para o sistema, no caso das Unidades Básicas de Saúde (UBS), o setor secundário integra os atendimentos médicos especializados, de suporte diagnóstico e terapêutico e serviço de urgência e emergência, e o setor terciário abrange os procedimentos de alta tecnologia e alto custo (Nascimento et al., 2017).

Segundo Portolese et al. (2017) levando em consideração a organização existente do SUS, o tratamento às pessoas com TEA ocorre principalmente nos níveis da $A B$ e da Atenção Especializada, seguindo o princípio da integralidade, abrangendo a rede de serviços ampliada, com a proposta da assistência nas áreas da saúde mental e geral, da educação/trabalho, da assistência social e a justiça. 
Apesar da relevância do assunto, ainda é escassa a produção de estratégias para o atendimento das pessoas com TEA no SUS (Lima et al., 2017).

Conforme relata Nascimento et al. (2017) a criança com TEA e sua família tem direito a cuidados integrais ofertados pelo SUS, pois além das necessidades próprias de sua condição, uma pessoa com TEA possui necessidades vitais comuns, como a vacinação, consultas, pré-natal, puericultura e saúde bucal.

Portolese et al. (2017) e Nascimento et al. (2017) descrevem os CAPS como setores de atendimento abertos e comunitários que devem propiciar atendimento de modo diário com uma assistência clinica individual e eficiente, viabilizando a inserção social do usuário, além de averiguar servir como base para a atenção à saúde mental na rede básica, administrar juntamente com o gestor local as ações de serviço de unidades hospitalares psiquiátricas e alimentar a lista de pacientes que fazem uso de fármacos em sua região.

Nascimento et al. (2017) ainda ressaltam que os CAPS funcionam com base na Lei 10.216/01, na Política Nacional de Saúde Mental e na Política Nacional de Proteção dos Direitos da Pessoa com TEA (Lei $n^{\circ}$ 12.764/12), proporcionando intervenções em saúde e tratamento para os usuários com variados tipos de transtornos psicossociais, objetivando a garantia do direito dos indivíduos em acompanhamento, de irem e virem livremente pelos serviços, comunidade e cidade.

Embora a Lei $n^{0} 12.764 / 12$ seja considerada uma grande conquista para as pessoas com TEA, em seus texto não se observa modalidades específicas de tratamento, mas recomenda diretrizes que tratam de pontos específicos como o cuidado integral as necessidades de saúde indispensáveis e próprias da pessoa com TEA, o diagnóstico precoce, atendimento com base multiprofissional, além de acesso a medicamentos e nutrientes (Oliveira et al., 2017).

Portolese et al. (2017) explica que a partir de 2002, os CAPS são vistos como fundamental instrumento especializado para 0 atendimento a pessoas com problemas de saúde mental severos, incluindo os TEA, onde nos casos específicos de crianças e adolescentes, foram criados os Centros de Atenção Psicossocial Infanto-juvenil (CAPSI). O CAPSI tem suas ações voltadas a infância e adolescência e se configura na estimulação da integração social e familiar das crianças e jovens, além de auxiliar no estabelecimento de suas autonomias. (Nascimento et al., 2017). 
O acompanhamento clínico executado no CAPSI envolve o cuidado diário e intensivo, sendo efetuado de forma personalizada e periódica, com o objetivo de atender necessidades particulares de cada paciente, contando com o auxílio de dispositivos como o Projeto Terapêutico Singular (PTS) e Técnico de Referência (TR), onde a equipe multiprofissional age de maneira transdisciplinar (Portolese et al., 2017).

\section{$O$ atendimento multiprofissional para os casos de autismo infantil}

Lima et al. (2017) narra que o atraso no tratamento de crianças com TEA ocorre na maioria dos casos, devido a insegurança por parte dos profissionais em diagnosticar, gerir e encaminhar à especialidades. As dificuldades que os usuários encontram podem dificultar a assistência de qualidade, como os obstáculos em detectar serviços que prestem cuidados a pessoas com TEA e a carência de profissionais com formação especializada em TEA (Portolese et al., 2017).

Guedes e Tada (2015) afirmam que a família é parte constituinte do suporte para o desenvolvimento da pessoa com TEA, juntamente com os cuidados multiprofissionais. A família, por auxiliar diretamente na educação e reabilitação a pessoa autista, necessita de atenção e auxilio adequado dos profissionais (Maia Filho et al. 2016).

Ainda que o DSM-V forneça critérios, o diagnóstico não é simples de ser realizado, cabendo aos profissionais responsáveis para este, serem submetidos a capacitações, uma vez que quanto mais cedo se observa traços autísticos, mas fácil será a reabilitação. A falta de conhecimento sobre o diagnóstico do autismo por parte dos profissionais, vem a intensificar a ideia de investimentos na capacitação dos profissionais da saúde, objetivando a melhora na qualidade da assistência (Guedes e Tada, 2015).

Portolese et al. (2017) descrevem que apesar das dificuldades encontradas, progressivamente, a assistência as pessoas com TEA tem sido direcionada ao CAPSI, beneficiando o curso da terapêutica, uma vez que esse local possui 
equipamentos e equipes multiprofissionais (médico pediatra, psicólogo, enfermeiros, terapeutas ocupacionais, fonoaudiólogos e assistentes sociais) qualificados na área.

Guedes e Tada (2015) explicam que não se tem conhecimento de um tratamento específico que envolva fármacos para o autismo, mas sim, para as condições interligadas a ele, o que em muitos casos, associado com acompanhamentos psicológicos, fisioterápicos, fonoaudiológicos, neurológicos, psiquiátricos e nutricionais, pode trazer efeitos benéficos, ocasionando uma diminuição considerável dos sintomas. O TEA consiste em um transtorno infindável, que independentemente do nível de gravidade, os indivíduos necessitam de acompanhamento interdisciplinar e especializado regular (Portolese et al. 2017).

Portolese et al. (2017) descreve que em sua pesquisa um resultado positivo no prognóstico, em pacientes que obtiveram o diagnóstico ao mesmo tempo em que eram assistidos por equipes multiprofissionais especializadas. A autora ainda ressalta que por mais que ações multiprofissionais apresentem ações eficientes, é necessário que cada profissional ponha em prática seus cuidados próprios da profissão, seguindo a mesma linha de atendimento pactuada com os demais profissionais.

\section{CONSIDERAÇÕES FINAIS}

O método escolhido para a terapêutica nos casos do TEA, deverá ser realizado de forma a envolver profissionais capacitados da rede, usuário e família com a finalidade de cuidar e envolver questões pertinentes a cada caso, mas que não será necessariamente definitivo, podendo sofrer variações e adequações em sua organização, conforme evolução do caso e surgimento de novas demandas e necessidades.

Para a $A B$, composta pelas UBS e suas equipes, fica a responsabilidade de identificar precocemente sinais do TEA e o encaminhamento para especialidades, além de oferecer cuidados contínuos ao paciente, uma vez que é considerada a 
ordenadora das RAS, estando em contato direto e primariamente com a comunidade.

Observa-se uma inexperiência da equipe multiprofissional da $A B$ em identificar, acompanhar e dar suporte ao usuário e família. Tal fato espelha a carência de políticas públicas voltadas para esses usuários no Brasil, que embora os atendimentos sigam a ideologia da assistência em rede, ainda faltam recursos para o cumprimento efetivo. O conhecimento cientifico profissional é o único meio de se garantir segurança e embasamento para tomada de decisões, tanto em relação a equipe multiprofissional com o paciente, quanto nas relações internas da própria equipe. Uma equipe multiprofissional capacitada, organizada e sistematizada constitui uma base segura para ações e cuidados eficientes.

\section{REFERÊNCIAS BIBLIOGRÁFICAS}

BRUNONI, D.; MERCADANTE, M. T.; SCHWARTZMAN, J. S. Transtornos do Espectro do Autismo. Clínica Médica: diagnóstico e tratamento. São Paulo: Atheneu, 2014.

CAMARGO, S. P. H.; BOSA, C. A. Competência social, inclusão escolar e autismo: revisão crítica da literatura. Psicologia e Sociedade. v. 21, n.1, p.65-74, 2009.

DEFENSE-NETVRAL, D. A.; FERNANDES, F. D. M. A oferta da terapia fonoaudiológica em locais de assistência a indivíduos com Transtornos do Espectro do Autista (TEA). Revista CoDAS. v. 28, n.4, p.59-462, 2016.

GUEDES, N. P. S.; TADA, I. N. C. A Produção Científica Brasileira sobre Autismo na Psicologia e na Educação. Revista Psicologia: Teoria e Pesquisa. Brasília, v.31, n.3, p.303-30, 2015.

KLIN, A. Autismo e síndrome de Asperger: uma visão geral. Revista Brasileira de Psiquiatria. v.28, n.1, p.3-11, 2006.

LIMA, R. C., COUTO, M. C. V., SOLIS, F. P., OLIVEIRA, B. D. C. \& DELGADO, P. G. G. Atenção psicossocial a crianças e adolescentes com autismo nos CAPSi da região metropolitana do Rio de Janeiro. Revista Saúde e Sociedade. v.26, n.1, p.196-207, 2017.

MAIA FILHO, A. L. M., MORAIS, L. A. N., SILVA, K. C. O. \& SANTIAGO, R. F. A importância da família no cuidado da criança autista. Revista Saúde em Foco. v.3, n.1, p.66-83, 2016.

MAIA, F. A., ALMEIDA, M. T. C., OLIVEIRA, L. M. M., OLIVEIRA, S. L. N., SAEGER, V. S. A., OLIVEIRA, V. S. D. \& SILVEIRA, M. F. Importância do acolhimento de pais que tiveram diagnóstico do transtorno do espectro do autismo de um filho. Cadernos de Saúde Coletiva. Rio de Janeiro, v.24, n.2, p.228-234, 2016.

NASCIMENTO, M. A., PEREIRA, M. \& GARCIA, S. C. M. Autismo infantil: acolhimento e tratamento pelo Sistema Único de Saúde. Revista Valore. v.1, n.2, p.155-167, 2017. 
OLIVEIRA, B. D. C., FELDMAN, C., COUTO, M. C. V. \& LIMA, R. C. Políticas para o autismo no Brasil: entre a atenção psicossocial e a reabilitação. Revista de Saúde Coletiva. v.27, n.3, p.707- 726, 2017. Organização Pan-Americana de Saúde. (2017). Folha informativa Transtorno do espectro autista. Disponível em https://www.paho.org/bra/index.php?ltemid=1098 Acesso em 07 de Abril de 2019. Organização Pan-Americana de Saúde. (2018). Classificação Estatística Internacional de Doenças e Problemas Relacionados à Saúde - CID-11. Disponível em: $\quad$ https://www.paho.org/bra/index.php?option=com_content\&view=article\&id=5702:omsdivulga- nova-classificacao-internacional-de-doencas-cid-11\&ltemid=875 Acesso em 20 de Fevereiro de 2019.

PEITER, C. C., SANTOS, J. L. G., LANZONI, G. M. M., MELLO, A. L. S. F., COSTA, M. F. B. N. A., ANDRADE, S. R. Redes de atenção à saúde: tendências da produção de conhecimento no Brasil. Escola Anna Nery. v.23, n.1, p.1-10, 2019.

PORTOLESE, J., BORDINI, D., LOWENTHAL, R., ZACHI, E. C. \& DE PAULA, C. S. Mapeamento dos serviços que prestam atendimento a pessoas com transtorno do espectro autista no Brasil. Cadernos de Pós-Graduação em Distúrbios do Desenvolvimento. v.17, n.2, p.79-91, 2017.

SOARES, C. B., HOGA, L. A. K., PEDUZZI, M., SANGALETI, C., YONEKURA, T. \& SILVA, D. R. A. D. Revisão integrativa: conceitos e métodos utilizados na enfermagem. Revista da Escola de Enfermagem. v.48, n.2, p.335-345, 2014.

ZAMPIROLI, W. C. \& SOUZA, V. M. P. Autismo infantil: uma breve discussão sobre a clínica e o tratamento. Revista Pediatria Moderna. v.48, n.4, p.126-130, 2012. 